\title{
Theranostic mRNA-loaded Microbubbles in the Lymphatics of Dogs: Implications for Drug Delivery
}

\author{
Heleen Dewitte ${ }^{*}$, Katrien Vanderperren ${ }^{2 *}$, Hendrik Haers², Emmelie Stock ${ }^{2}$, Luc Duchateau ${ }^{3}$, Myriam \\ Hesta $^{4}$, Jimmy H. Saunders ${ }^{2}$, Stefaan C. De Smedt ${ }^{1 凶}$, and Ine Lentacker ${ }^{1}$ \\ 1. Laboratory for General Biochemistry and Physical Pharmacy, Faculty of Pharmacy, Ghent University, Ghent, Belgium; \\ 2. Department of Veterinary Medical Imaging and Small Animal Orthopaedics, Faculty of Veterinary Medicine, Ghent University, \\ Merelbeke, Belgium; \\ 3. Department of Comparative Physiology and Biometrics, Faculty of Veterinary Medicine, Ghent University, Merelbeke, Belgium; \\ 4. Department of Animal Nutrition, Faculty of Veterinary Medicine, Ghent University, Merelbeke, Belgium. \\ * both first authors contributed equally to this work.
}

$\triangle$ Corresponding author: Stefaan C. De Smedt: Laboratory for General Biochemistry and Physical Pharmacy, Ottergemsesteenweg 460, B-9000 Ghent, Belgium. Tel +32 926480 76; Fax +32 926481 89; Email: Stefaan.DeSmedt@UGent.be.

( ) Ivyspring International Publisher. This is an open-access article distributed under the terms of the Creative Commons License (http://creativecommons.org/ licenses/by-nc-nd/3.0/). Reproduction is permitted for personal, noncommercial use, provided that the article is in whole, unmodified, and properly cited.

Received: 2014.08.08; Accepted: 2014.09.22; Published: 2015.01.0I

\begin{abstract}
Microbubbles have shown potential as intralymphatic ultrasound contrast agents while nanoparticle-loaded microbubbles are increasingly investigated for ultrasound-triggered drug and gene delivery. To explore whether mRNA-nanoparticle loaded microbubbles could serve as theranostics for detection of and mRNA transfer to the lymph nodes, we investigate the behavior of unloaded and mRNA-loaded microbubbles using contrast-enhanced ultrasound imaging after subcutaneous injection in dogs. Our results indicate that both types of microbubbles are equally capable of rapidly entering the lymph vessels and nodes upon injection, and novel, valuable and detailed information on the lymphatic structure in the animals could be obtained. Furthermore, additional observations were made regarding the dynamics of microbubble lymph node uptake. Importantly, neither the microbubble migration distance within the lymphatics, nor the observed contrast signal intensity was influenced by mRNA-loading. Although further optimization of acoustic parameters will be needed, this could represent a first step towards ultrasound-guided, ultrasound-triggered intranodal mRNA delivery using these theranostic microbubbles.
\end{abstract}

Key words: Contrast-enhanced ultrasound imaging, mRNA, microbubbles, mRNA-loaded microbubbles, dogs, lymph nodes.

\section{Introduction}

Over the years, microbubbles have gained popularity as contrast agents for ultrasound imaging. When injected intravenously, these microbubbles remain within the vasculature where they increase the echogenicity of blood. In this way, enhanced contrast between blood and soft tissues can be achieved. This has led to the FDA and EMEA approval of various microbubble contrast agents for echocardiography (e.g. Definity ${ }^{\mathrm{TM}}$, SonoVue ${ }^{\mathrm{TM}}$, Sonazoid ${ }^{\mathrm{TM}}$ and Opti- son $^{\mathrm{TM}}$ ) [1]. Ever since, new applications for these contrast agents are under investigation, including their potential to enhance contrast in the lymphatics. For this, the microbubbles are injected intradermally (i.d.) or subcutaneously (s.c.), after which they drain to the lymphatics and accumulate in the lymph nodes. This is particularly interesting, since studies in tumor-bearing animals as well as in human breast cancer patients showed that peritumoral i.d. and s.c. in- 
jection of microbubbles results in their drainage to the sentinel lymph node (SLN) [2-7]. This lymph node is especially important, as it is the first node to come in contact with tumor material, making it most vulnerable to metastasis. Therefore, SLN detection, biopsy and removal are routine procedures in oncology to detect and remove disseminated tumor cells [8,9]. Currently used standard methods for SLN detection are the injection of blue dyes which aid to localize the SLN visually during surgery, or via scintigraphy after injection of radiolabeled dyes $[8,10]$. However, these procedures both come with their limitations, resulting in an ongoing search for novel SLN mapping modalities, such as microbubble contrast-enhanced ultrasound imaging (CEUS), which could represent a non-radioactive, non-invasive and potentially more patient-friendly alternative $[11,12]$.

In addition to these clinical applications, microbubbles are also under preclinical investigation as potential drug- and gene-delivery agents. When microbubbles are exposed to ultrasound, their gas core starts cavitating, i.e. it continuously expands and shrinks. When the amplitude of the applied ultrasound waves is augmented this cavitation can become unstable, eventually causing the microbubbles to implode. Both stable cavitation as well as microbubble implosion can affect neighboring cells by either stimulating endocytosis or by the formation of temporary pores in the cell membranes (i.e. sonoporation) [13]. Both mechanisms can be used for ultrasound-triggered delivery of drugs and genes in vitro and in vivo [14-16].

Based on this knowledge, we recently reported on the design of microbubbles that can be loaded with mRNA-nanoparticles and have potential applications in cancer immunotherapy [17, 18]. For this, we showed that such mRNA-loaded bubbles could be used to induce transient protein-expression in murine dendritic cells (DCs). These cells are important targets in immunotherapy, as they are the key initiators of antigen-specific immune responses [19]. Therefore, by introducing tumor antigen expression in DCs, these cells can present fragments of these tumor antigens to $\mathrm{T}$ cells, leading to antigen-specific $\mathrm{T}$ cell activation [19-21]. In this way, the immune system can be stimulated to selectively recognize and destroy cancer cells [18]. Although the clinical results are encouraging, DC-based vaccination still needs to overcome a number of hurdles, one of which is the efficient delivery of antigenic material to the cells [22]. More specifically, current methods in the production of DC-based vaccines still focus on the ex vivo loading of these cells with tumor antigens, which makes DC-based immunotherapy time-consuming, patient-specific and expensive $[23,24]$. Thus, there is an unmet need for methods that could deliver antigens to DCs in vivo. For this, the anatomical sites of interest would be lymphoid organs, such as the lymph nodes, as these harbor large numbers of DCs $[25,26]$. Therefore, we designed microbubbles that can be loaded with antigen mRNA, and were shown to efficiently transfect DCs in vitro [17]. Moreover, when these mRNA-sonoporated DCs were injected as vaccines in tumor-bearing mice, this resulted in the induction of potent antitumor immune responses, even leading to complete tumor regression and long-term immunological protection against tumor regrowth in $30 \%$ of the vaccinated animals [18]. Taken together, this strategy has shown promise in vitro, and could also have potential for immediate in vivo applications. More specifically, if these microbubbles could reach the lymphatics after s.c. injection, they could serve as interesting agents for the ultrasound-guided and ultrasound-triggered antigen delivery to intranodal DCs.

To evaluate whether the proposed mRNA-loaded microbubbles could be used for this purpose, we investigated if indeed these nucleic-acid loaded bubbles can reach the lymph nodes after s.c. injection, and if this can be monitored with CEUS. For this, we performed a cross-over study where both unloaded and mRNA-loaded microbubbles were injected subcutaneously in dogs. Using CEUS, we determined (a) which injection site resulted in optimal lymphatic microbubble appearance, $(b)$ if there were differences in kinetics and lymph node contrast-enhancement for both types of microbubbles and (c) to what extent the nucleic acid-loaded microbubbles could have potential as intralymphatic theranostics.

\section{Materials and methods mRNA and mRNA-lipoplexes}

Luciferase mRNA was produced by in vitro transcription from pBlue-Luc-A50 plasmids. The plasmids were purified using a QIAquick PCR purification kit (Qiagen, Venlo, The Netherlands) and linearized using Dra I restriction enzymes (Promega, Leiden, The Netherlands). Linearized plasmids were used as templates for the in vitro transcription reaction using the T7 mMessage mMachine kit (Ambion, Life Technologies, Ghent, Belgium). The resulting capped and polyadenylated mRNAs were purified by DNase I digestion, $\mathrm{LiCl}$ precipitation and washed with $70 \%$ ethanol. The mRNA concentration was determined by measuring the absorbance at $260 \mathrm{~nm}$. mRNA was stored in small aliquots at $-80^{\circ} \mathrm{C}$ at a concentration of $1 \mu \mathrm{g} \mu \mathrm{l}^{-1}$.

mRNA lipoplexes were prepared by complexing 
the mRNA to cationic liposomes. For this, liposomes were prepared consisting of $48.75 \%$ DOTAP $(1,2-$ dioleoyl-3-trimethylammonium-propane), $\quad 48.75 \%$ DOPE (1,2-dioleoyl-sn-glycero-3-phosphoethanolamine, both Avanti Polar Lipids) and 2.5\% DSPE-PEG3400-biotin [1,2-distearoyl-sn-glycero-3phosphoethanol-amine-N-[biotinyl(polyethyleneglycol)-3400] (Laysan Bio Inc, Arab, Alabama). Fluorescently labeled mRNA-lipoplexes were prepared by incorporating $1 \%$ Cholesteryl-BODIPY ${ }^{\circledR}$ FL $\quad$ C12 (Avanti Polar Lipids). The appropriate amounts of lipids (dissolved in chloroform) were transferred to a round-bottom flask, and the chloroform was evaporated under nitrogen. Subsequently, the resulting lipid film was hydrated in RNase-free water (Ambion) to obtain a final lipid concentration of $1 \mathrm{mg} \mathrm{ml}^{-1}$. The resulting cationic liposomes were sonicated for $15 \mathrm{~min}$ in a bath sonicator (Branson Ultrasonics, Dansbury, USA). Then, the cationic liposomes were mixed with mRNA in OptiMem ${ }^{\circledR}$ (Gibco Invitrogen) to obtain mRNA-lipoplexes at a cationic lipid-to-mRNA charge $(\mathrm{N} / \mathrm{P})$ ratio of 8.

\section{Unloaded and mRNA-loaded microbubbles}

Lipid microbubbles loaded with mRNA-lipoplexes were prepared as described previously [17]. Briefly, perfluorobutane (F2 chemicals, Preston, UK) microbubbles stabilized by a lipid coat consisting of DPPC (1,2-dipalmitoyl-sn-glycero3-phosphocholine) (Lipoid, Ludwigshafen, Germany) and DSPE-PEG3400-biotin [1,2-distearoyl-sn-glycero3-phosphoethanolamine-N-[biotin(polyethylene glycol)-3400] in a 85:15 molar ratio were prepared as follows. Appropriate aliquots of both lipids, dissolved in chloroform, were transferred to a round bottom flask. After chloroform evaporation, the lipids were dissolved in a 1:2:7 glycerol-propyleneglycol- $\mathrm{H}_{2} \mathrm{O}$ mixture to obtain a clear solution with a final concentration of $4.6 \times 10^{-4} \mathrm{mmol} \mathrm{ml}^{-1}$. Aliquots of this lipid solution were transferred to $2.5 \mathrm{ml}$ chromatography vials and the vial headspace was filled with perfluorobutane gas (F2 chemicals, Preston, UK). In order to form microbubbles, the vials were shaken at high-frequency in a Capmix ${ }^{\mathrm{TM}}$ device (3M-ESPE, Diegem, Belgium) for 15s. Microbubbles were avidinylated by first performing washing steps to remove excess lipids, after which the microbubbles were incubated with avidin (Cell Sciences, Canton, USA) for $5 \mathrm{~min}$. Then, the bubbles were washed to remove excess avidin and the final bubble cake was redispersed in a sterile, endotoxin- and nuclease-free $5 \%$ glucose solution (Sigma-Aldrich, Diegem, Belgium). $5 \mathrm{~min}$ prior to injection, $150 \mu \mathrm{l}$ avidinylated bubbles were mixed with either $100 \mu 1$ OptiMem $^{\circledR}$ (for the unloaded bubbles) or $100 \mu \mathrm{l}$ mRNA-lipoplexes (for the mRNA-loaded microbubbles, corresponding to $10 \mu \mathrm{g}$ mRNA per injection).

\section{Microbubble characterization}

The size distribution and concentration of mRNA-loaded microbubbles was determined via coulter counter measurements using a Beckman-coulter Multisizer 4 (Analis SA, Suarlée, Belgium). Confocal microscopy was performed to evaluate the loading of the microbubbles with fluorescently labeled mRNA-lipoplexes. This was done using a Nikon C1si confocal laser scanning module attached to a motorized Nikon TE2000-E inverted microscope (Nikon, Brussels, Belgium), and a Plan Apo 60X 1.4 NA oil immersion objective lens (Nikon).

\section{Dogs}

Six healthy research beagles ( 3 spayed females, 1 intact female and 1 castrated male and 1 intact male) were used in this experiment. All procedures were performed in accordance with the Ethical committee guidelines of the Ghent University (2014/31). The mean age of the animals was 7 years. Body weight ranged from $9.7 \mathrm{~kg}$ to $15.4 \mathrm{~kg}$ with a mean of $10.8 \mathrm{~kg}$. Dogs were healthy based on physical findings and routine laboratory data.

\section{Contrast-enhanced ultrasound imaging}

For CEUS experiments, the animals were sedated by intravenous injection of a combination of $\mathrm{Bu}-$ torphanol (Dolorex ${ }^{\circledR}, 0.4 \mathrm{mg} \mathrm{kg}^{-1}$ ) and Acepromazine (Placivet $^{\circledR}, 0.04 \mathrm{mg} \mathrm{kg}^{-1}$ ). Once sedated, the animals were manually restrained in dorsal recumbency and received a subcutaneous injection of microbubbles. First, different injection sites in the caudal abdomen and inguinal region were evaluated based on contrast migration to choose the optimal location for further experiments. After injection of $250 \mu \mathrm{l}$ microbubbles, the contrast timer was started and the injection site was massaged during $1 \mathrm{~min}$. After this, continuous CEUS imaging was performed with contrast-specific software using a 12-5 MHz linear transducer (L12-5) of a Philips iU-22 US scanner (Philips Medical systems, Bothell, Wa) for at least $6 \mathrm{~min}$. Mechanical index (MI) was set at a low level $(\mathrm{MI}=0.08)$ to achieve microbubble resonance with production of harmonic frequencies. Machine settings such as the overall gain (80\%), time gain compensation, depth $(2.5 \mathrm{~cm})$, frame rate $(10 \mathrm{~Hz})$ persistence (off) and dynamic range were set at the same value for every examination. Only one focal spot was used, and was set at the lowest level of the image. Destruction of intranodal microbubbles was performed at the end of the imaging period by setting the acoustic power at the highest level $(\mathrm{MI}=0.61)$. Multiple subsequent bursts were delivered 
until the echo intensity stabilized.

This study was performed using a crossover design, with all dogs receiving 2 injections of both unloaded and mRNA-loaded microbubble formulations, on two injection sites (in the left and right abdominal region) at 2 different time points separated by a 2 week wash-out period, resulting in a total of 24 injections (12 with unloaded bubbles and 12 with mRNA-loaded bubbles, Table 1).

Table I: Design of the crossover CEUS study.

\begin{tabular}{|c|c|c|}
\hline Day & Dogs & Microbubble injections \\
\hline Week 1, day 1 & $1,2,3$ & $\begin{array}{l}\text { Left: unloaded microbubbles } \\
\text { Right: mRNA-loaded microbubbles }\end{array}$ \\
\hline Week 1, day 2 & $4,5,6$ & $\begin{array}{l}\text { Left: mRNA-loaded microbubbles } \\
\text { Right: unloaded microbubbles }\end{array}$ \\
\hline \multicolumn{3}{|l|}{2 week wash-out } \\
\hline Week 3, day 1 & $1,2,3$ & $\begin{array}{l}\text { Left: mRNA-loaded microbubbles } \\
\text { Right: unloaded microbubbles }\end{array}$ \\
\hline Week 3, day 2 & $4,5,6$ & $\begin{array}{l}\text { Left: unloaded microbubbles } \\
\text { Right: mRNA-loaded microbubbles }\end{array}$ \\
\hline
\end{tabular}

\section{Image analysis}

Image analysis was performed using QLAB quantification software (Philips) and ImageJ. Microbubble migration distances were calculated as a linear distance between the injection site and the most distant microbubble contrast signal. Measurements were performed by two independent, blinded observers. Maximal echo intensities were measured using Image $J$ at different timepoints after injection on a region of interest (ROI) within the lymphatics (i.e. within the enhanced lymph nodes or within the lymph vessels when no lymph node enhancement could be observed). Analysis of burst destruction of intranodal microbubbles was performed by drawing a ROI over the lymph node of interest, after which the mean echo intensity was studied as a function of time using the QLAB quantification software. In the figures, time is indicated in min:s on the CEUS images, and the depth scale bar is shown at the right-hand side of the corresponding B-mode images (the distance between two ticks is $5 \mathrm{~mm}$ in all images).

\section{Statistical analysis}

The migration distance and the maximal echo intensity was compared between the loaded and unloaded bubbles by a mixed model with dog as random effect and period and bubble type as categorical fixed effects, using the F-test at the 5\% significance level. The contrast echo intensity over time was compared between the loaded and unloaded bubbles by a mixed model with dog as random effect and period, time, bubble type and the interaction between time and bubble type as categorical fixed effects, using the
F-test at the 5\% significance level.

Within and between observer variability was determined by estimating the within and between observer variances for a same assessment (i.e., the same dog, side, bubble type and period) by the restricted maximum likelihood procedure (REML). These estimated variances were next used to determine the range in which $95 \%$ of the differences between two measurements of the same observers and two different observers are contained. SAS version 9.3 was used for all analyses.

\section{Results}

\section{Characterization of unloaded and mRNA-loaded microbubbles}

mRNA-lipoplex loaded microbubbles were prepared by first complexing the mRNA to cationic liposomes, to form mRNA-lipoplexes in which the mRNA is protected against degradation (Additional file 5: Figure S1). These mRNA-lipoplexes are then attached to the surface of avidinylated lipid microbubbles, as shown in Figure 1A. To demonstrate effective loading, we performed confocal microscopy on microbubbles that were loaded with fluorescently labeled mRNA-lipoplexes. As demonstrated in Figure 1B, the mRNA-lipoplexes are clearly present around the microbubble surface, and we did not observe any aggregation. The size and concentration of the microbubble preparations was determined via coulter counter measurements. The unloaded microbubbles had a mean number diameter of $2.48 \pm 1.57 \mu \mathrm{m}$. Attachment of mRNA-lipoplexes did not significantly alter the mean diameter $(1.93 \pm 1.25 \mu \mathrm{m})$ (Figure 1C). The concentration of the microbubble preparations was $2.68 \pm 0.86 \times 10^{9}$ bubbles $\mathrm{ml}^{-1}$.

\section{Factors influencing intralymphatic CEUS}

Based on previous experiments studying the lymphatic drainage of SonoVue ${ }^{\mathrm{TM}}$ microbubbles by Goldberg et al. [7], we performed subcutaneous injections of both unloaded and mRNA-loaded microbubbles in dogs. When performing these experiments, we observed that a number of factors influenced the CEUS signals.

First of all, we noticed important differences in the migration of the microbubbles depending on the location of the injection site. When injecting the microbubbles in the groin, close to the inguinal lymph node, we could not observe any lymphatic uptake of the contrast agents. The reasons for this are probably dual. First of all, lymphatic uptake occurs through a passive process that depends on the interstitial pressure at the injection site. Hence, regions such as the 
groin, where the skin is loose and the interstitial pressure is low, are not preferable [27]. In addition to pressure-related differences, some tissues, such as the mammary regions of female dogs, contain a more developed lymph vessel network. In accordance to this, initial s.c. injections of the contrast agents in the loose skin of the abdominal region did not result in migration of the contrast agents from the injection site. This despite the fact that the microbubbles were injected at a distance of merely $20 \mathrm{~mm}$ from the inguinal lymph node, as indicated by palpation and B-mode ultrasound imaging. On the other hand, injection around the dog's nipple resulted in extensive microbubble drainage in all female dogs, as shown in Figure 2. Migration from the injection site was seen for all injections with both unloaded $(8 / 8)$ and mRNA-loaded (8/8) microbubbles in the female dogs, and after most of the injections around the nipples of the male dogs (4/4 for the unloaded bubbles and 2/4 for the mRNA-loaded bubbles). This is not unexpected, as the mammary regions accommodate a vast network of lymph vessels that lead to multiple clusters of lymph nodes close to the mammary glands, as well as more distant gastric and mesenteric lymph nodes, depending on the location of the mammary gland (e.g. axillary versus inguinal mammary glands) [28]. Based on these observations, further injections were performed s.c. around the left and right nipple of the inguinal mammary gland.
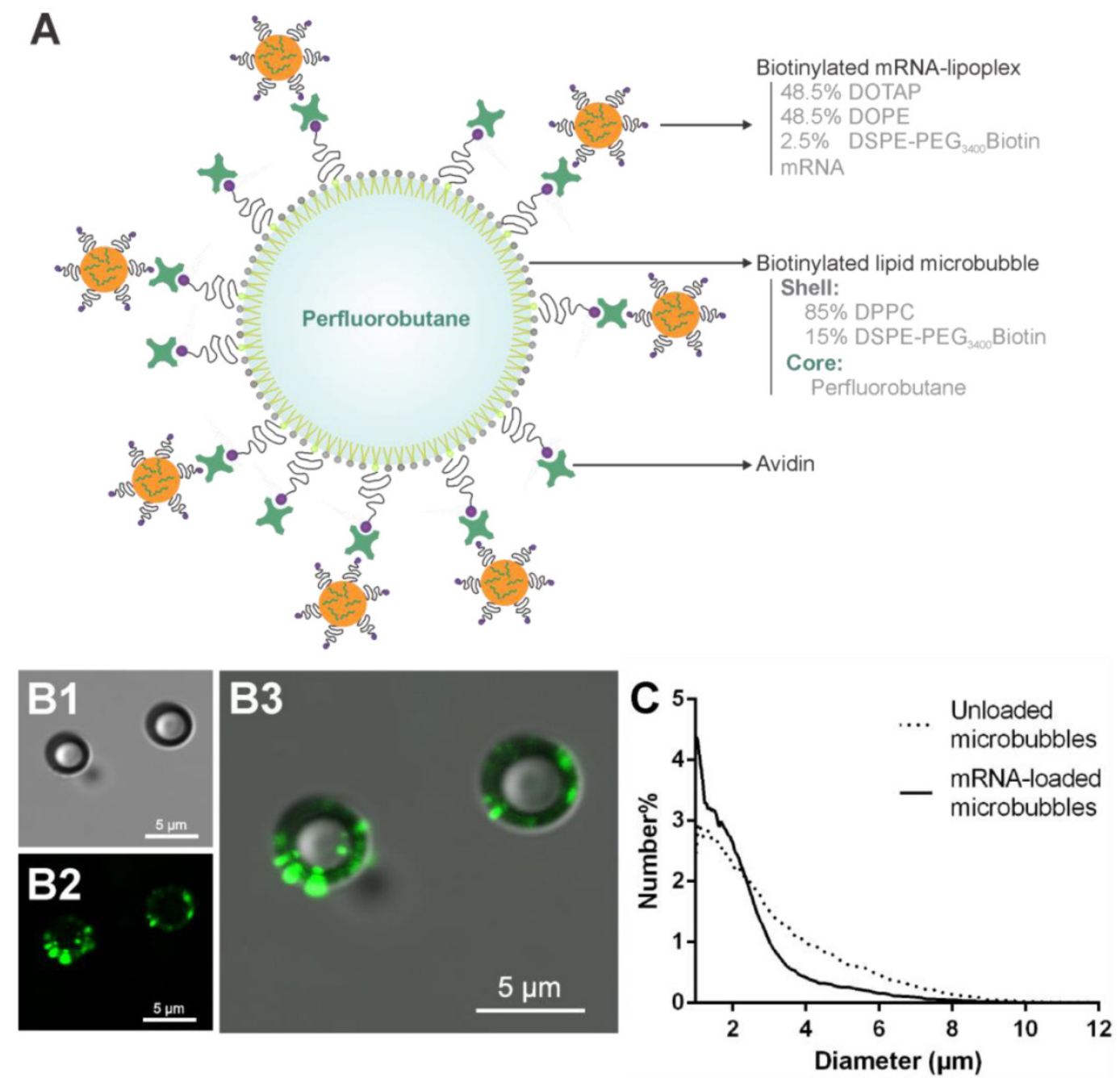

Figure I: Characterization of mRNA-loaded microbubbles. (A) Graphical representation of the mRNA-loaded microbubble composition. (B) Confocal microscopic images of microbubbles loaded with fluorescently labeled mRNA-lipoplexes: (BI) transmission, (B2) green fluorescent mRNA-lipoplexes, (B3) overlay. (C) Size distribution of unloaded and mRNA-loaded microbubbles. 

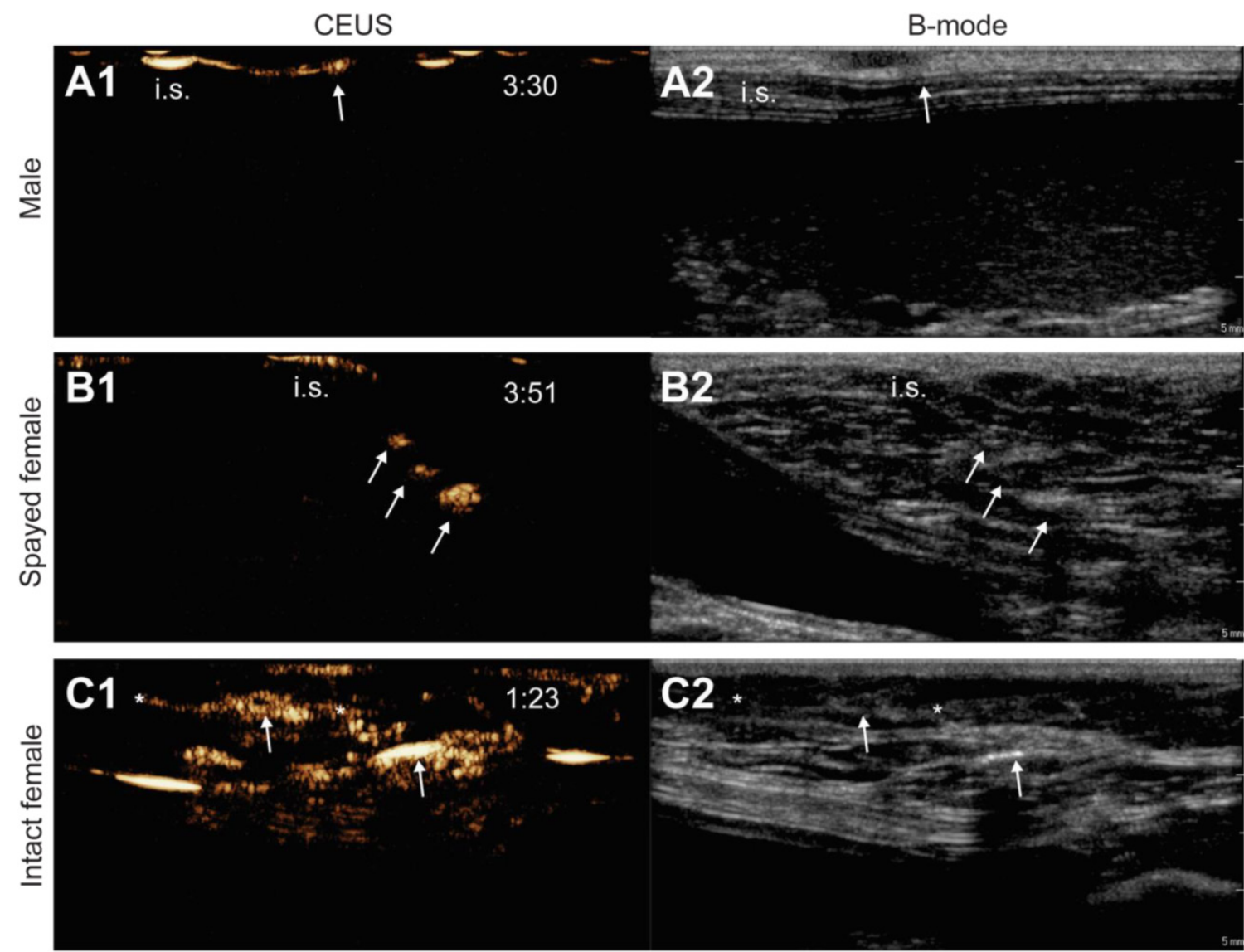

Figure 2: Influence of animal properties on the lymphatic CEUS images. Pictures show the CEUS images obtained after s.c. injection of unloaded microbubbles around the mammary glands of a male $\operatorname{dog}(\mathrm{AI})$, a spayed female $\operatorname{dog}(\mathrm{BI})$ and an intact female $\operatorname{dog}(\mathrm{CI})$. Respective B-mode images are shown in A2, $\mathrm{B} 2$ and $\mathrm{C2}$. The injection site is indicated as "i.s." (the injection site for images $\mathrm{Cl}$ and $\mathrm{C} 2$ is out of the field-of-view), arrows indicate the accumulation of microbubbles in the lymph nodes and lymph vessels are pointed out with asterisks. Time after microbubble injection is noted on the CEUS images (in min:s).

Besides the effects of the injection site, inter-animal variation in the mammary gland lymphatic network also played a role. In male dogs, the mammary glands are markedly less developed compared to females, which also results in a less dense lymphatic network and fewer intramammary nodes. Moreover, the male dogs clearly showed a less thick subcutaneous fat layer (i.e. where the lymph vessels and nodes are located) compared to all females, as evidenced by the B-mode images in Figure 2, images $\mathrm{A} 2$, B2 and $\mathrm{C} 2$. As a result, contrast agent migration after injection was less pronounced in the males compared to the females. In the intact male, injection next to the testicles resulted in more distant microbubble migration when compared to injection around the mammary glands, which therefore represented a valuable alternative to injection around the mammary glands. Beside these gender-related factors, we also observed differences between spayed and intact females. After microbubble injection in the intact female, the lymphatic structures were visible as a vast network with multiple nodes and vessels extending more deeply into the subcutaneous fat pad (Figure 2, images $\mathrm{C} 1$ and $\mathrm{C} 2$, and Additional file 1: supplementary video 1 , observed for all injections with both unloaded and mRNA-loaded microbubbles). Such a branched lymph network could not be observed in any of the 3 spayed females.

Of course, this inter-animal variation makes it more difficult to fully exclude anatomical bias when comparing the lymphatic drainage of unloaded and mRNA-loaded microbubbles. The cross-over design used for this study, where all dogs receive s.c. injections with both unloaded and mRNA-loaded microbubbles in the nipples of both the left and the right caudal mammary gland, was used to take this variability into account and allow a better comparison of both microbubble types.

\section{Lymphatic network visualization}

After microbubble injection and massaging of the injection site, the migration of the contrast agent was observed unidirectional, with the contrast agents leaving the injection site at only one end in the spayed females and the males, as exemplified by Figure 3A. In the intact female, microbubble migration occurred both cranially as well as caudally. The migrating contrast agents were followed through the lymph vessels 
towards draining lymph nodes (Figure 3, Images B1, B2, C1 and C2, and Additional file 2: supplementary video 2). Importantly, in none of the injections $(0 / 24)$ did we observe blood pool contrast enhancement, indicating that the microbubbles were restricted to the interstitium, the lymph vessels and the lymph nodes. Moreover, when the contrast agents were injected on one side (either left or right) of the animal, contrast signal was only observed on that same side of the animal. We did not observe contrast crossing over the midline of the dogs in any of the cases. One or multiple lymph nodes could be identified using CEUS after 9/12 injections of both unloaded and mRNA-loaded microbubbles, and the size of the enhanced lymph nodes ranged from 1.6 to $7.0 \mathrm{~mm}$.

Interestingly, CEUS imaging revealed rather detailed information on the lymphatics' anatomy: in accordance to previous reports, CEUS could provide information on the location of the lymph nodes, the number of afferent and efferent lymph vessels [5]. In addition, we could observe different patterns of contrast agent presence within the nodes. Some lymph nodes, such as the one shown in Figure 4A, are completely filled with contrast agent, whereas others rather exhibit a "hollow" appearance, with contrast material only appearing at the outer rim of the node, as shown in Figure 4, images B1, B2, C1 and C2. This can be explained by the fact that afferent lymph vessels can either directly discharge their content into the draining lymph nodes, or the afferent lymph vessels run through or over the nodes, without effectively discharging the lymph within the node [29].
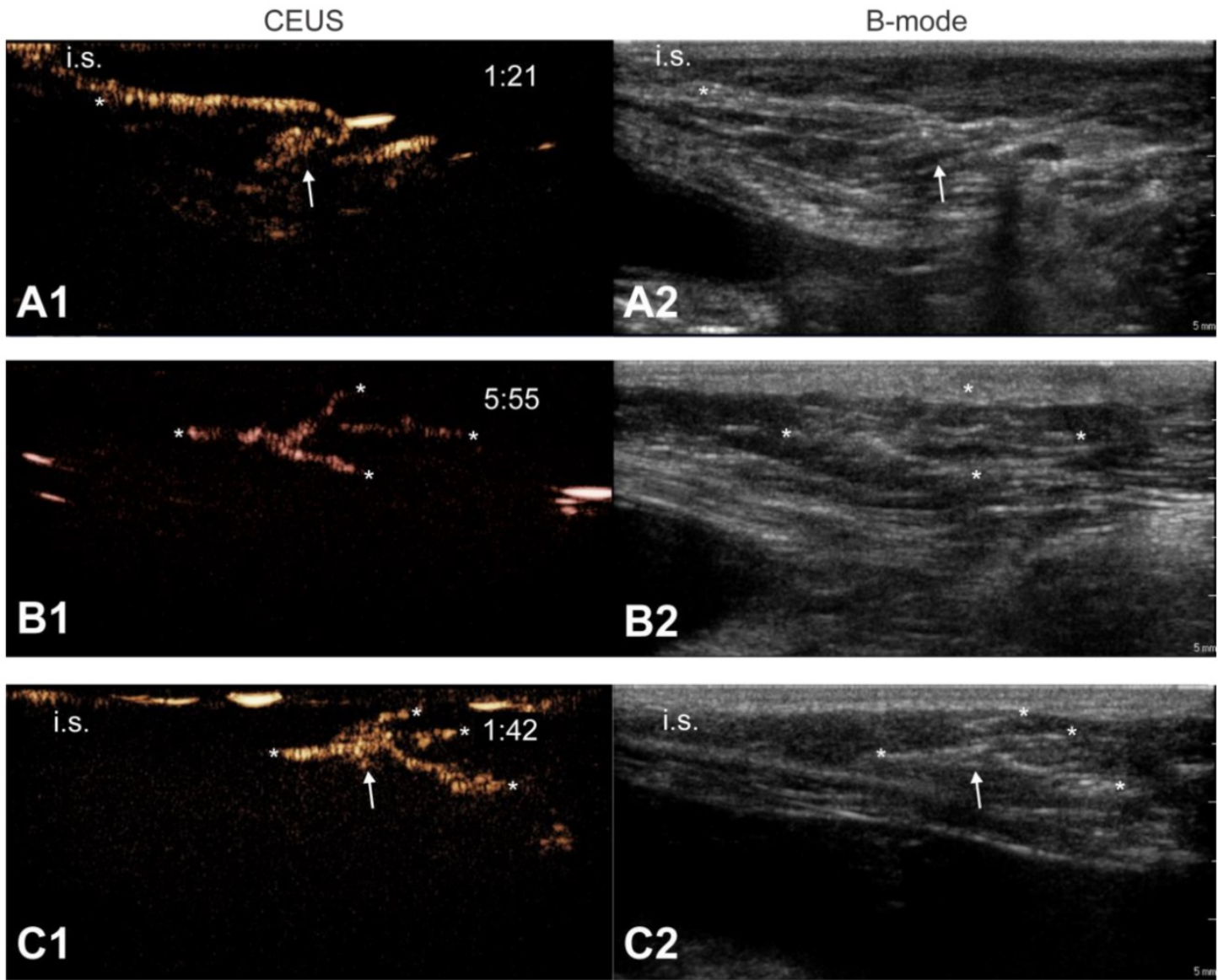

Figure 3: Contrast agent migration from the injection site into the lymph vessels and nodes. Upon microbubble injection, unidirectional transport of microbubbles away from the injection site, through an afferent lymph vessel into a draining lymph node can be observed with CEUS (AI). Image (BI) shows the trafficking of microbubble contrast signal through branched lymph vessels. In (CI), the CEUS image shows a lymph node connected to one afferent lymph vessel and 3 efferent lymph vessels that take the contrast agents further away from the injection site. Corresponding B-mode images are shown in (A2), (B2) and (C2), respectively. Images were obtained after injection of mRNA-loaded microbubbles in different female dogs. The injection site is marked as "i.s." (unless outside of the field-of-view), arrows indicate lymph nodes and asterisks point out lymph vessels. Time after microbubble injection is noted on the CEUS images (in min:s). 


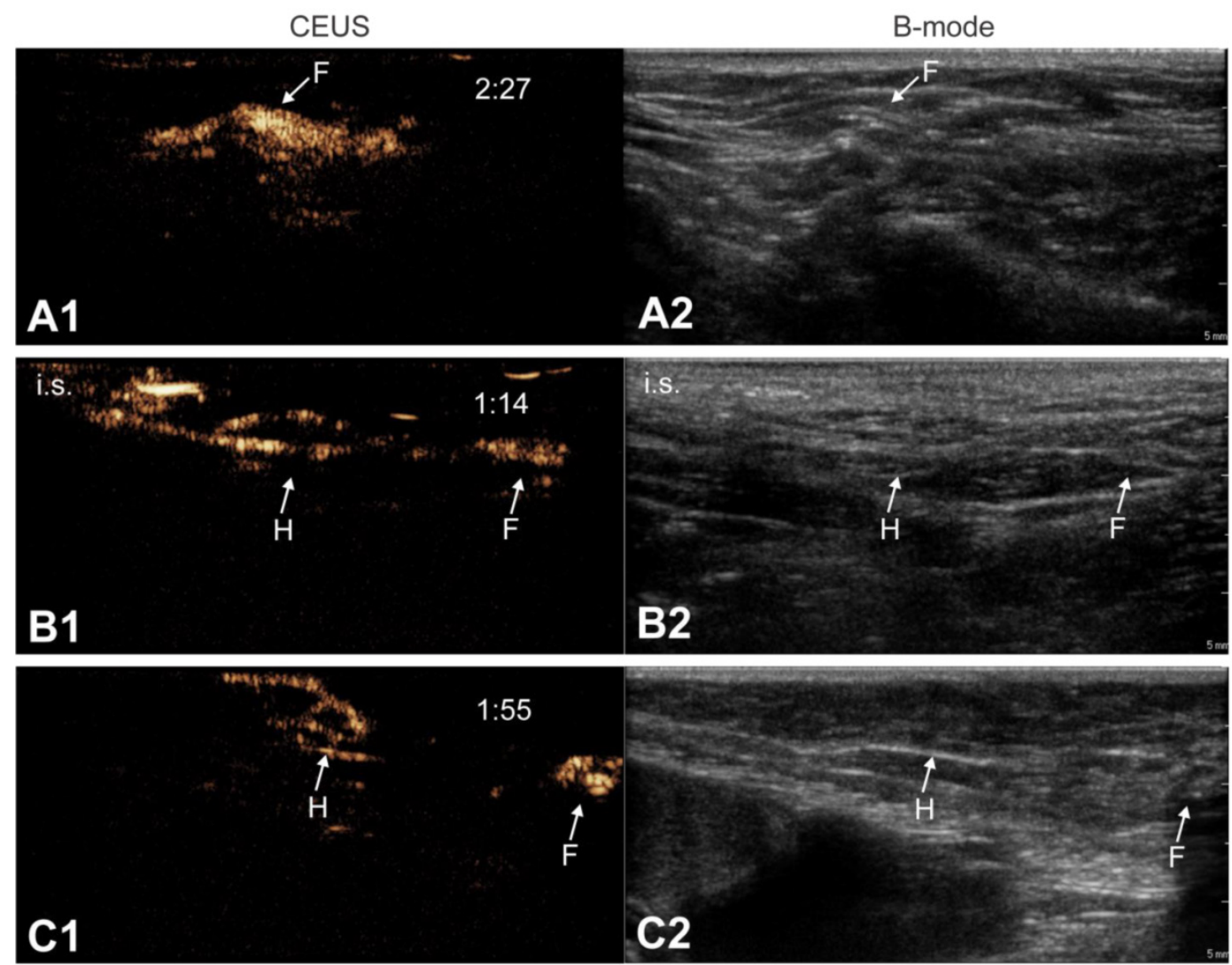

Figure 4: Lymph node anatomy observed by CEUS. CEUS can be used to identify the relation between afferent lymph vessels and draining lymph nodes. Lymph vessels either distribute their content within the lymph node ("filled" nodes, AI-A2) or they go around the lymph nodes without discharging its contents into the node ("hollow" nodes, $\mathrm{BI}-\mathrm{B} 2$ and $\mathrm{Cl}-\mathrm{C} 2$ ). In the latter scenario, we always observed a node with a "hollow" appearance (indicated as "H"), followed by a more distant "filled" node (indicated as "F"). Images were obtained from 3 different animals. Where possible, the injection site is pointed out as "i.s.". Time after microbubble injection is noted on the CEUS images (in min:s).

\section{Lymphatic CEUS using mRNA-loaded microbubbles versus unloaded microbubbles}

In this study, we compared the CEUS images after s.c. injection of unloaded and mRNA-loaded microbubbles, using three main scoring criteria: (a) the migration distance of the contrast agents from the injection site within the lymphatics, (b) the maximal intensity of the contrast signal and (c) the stability of the contrast agents in the lymph vessels and nodes over time.

With regards to the first parameter, the migration distance, we could detect microbubble migration from the injection site for all injections with unloaded microbubbles (12/12), and in 10/12 cases after mRNA-loaded microbubble injections. The 2 injections that did not result in contrast agent drainage were observed in the male dogs ( 1 in the castrated male and 1 in the intact male). The microbubble migration distance (calculated linearly from the injection site) was on average $24.7 \pm 13.7 \mathrm{~mm}$ for unloaded microbubbles and $29.0 \pm 19.0 \mathrm{~mm}$ for mRNA-loaded microbubbles (Figure 5A). No significant difference was observed for migration distance between the loaded and unloaded bubbles, with a mean difference equal to 4.33 (95\% CI: [-4.55; 13.22]). It should be noted that even though these results were analyzed based on injections of unloaded and mRNA-loaded microbubbles within the same mammary gland, variation based on the exact injection site cannot be excluded, even though injections were always performed caudally from the nipple by the same person. For instance, it was shown by Goldberg et al. [6] that injection sites that were merely $1 \mathrm{~cm}$ apart in distance, could result in drainage to a different lymph node, which can obviously impact the microbubble migration distance that was observed. As the exact injection site was not marked at the time of the first imaging session, variation related to the injection site location cannot be fully excluded. The variation between repeated observations of the same observer was 23.9, and the extra variation due to different observers was 9.0 for loaded bubbles, leading to somewhat larger 95\% intervals for differences between two measurements of two different observers as compared to the same observer. On the other hand, for the unloaded microbubbles, the variation due to repeated observations of the same observer was 16.7, and there was no extra variation due to different observers, resulting in the same $95 \%$ intervals for differences between two measurements of two different observers as compared 
to the same observer (Additional file 5: Figure S2).

In addition to distance measurements, we compared the maximal echo intensity that we could obtain within the lymphatics after injection of unloaded and mRNA-loaded microbubbles. As for the migration distance, we could observe no significant differences in lymphatic contrast enhancement between both types of bubbles (Figure 5B), with a mean difference between loaded and unloaded bubbles equal to -2.56 (95\% CI:[-15.04; 9.91]). Moreover, when the contrast echo intensity was evaluated as a function of time, we could observe relatively long-term contrast agent stability within the lymphatics, as in all cases where contrast migration was observed, the microbubbles could still be clearly detected $6 \mathrm{~min}$ after microbubble injection (Additional file 3: Supplementary video 3). There was merely a slight decay of the echo intensity over this period, and we could not detect significant differences between both types of microbubbles (Figure 6).

Keeping in mind the final aim of the mRNA loaded microbubbles, namely the ultrasound-triggered delivery of mRNA to DCs that reside within the lymph nodes, we tested whether intranodal mRNA-loaded microbubbles could indeed be imploded by applying higher-intensity ultrasound bursts. For this, we used the scanner's preset burst function, and looked at the decrease in mean echo contrast intensity within the lymph node. As shown in Figure 7, with each burst, the microbubble echo intensity was reduced, reaching a minimum after 6 bursts. A video of this burst destruction can be found in supplementary data (Additional file 4: supplementary video 4 ).

\section{Discussion}

In recent years, drug- and gene loaded microbubbles emerged as interesting theranostics after intravenous injection. This, however, is the first report on the development of theranostic mRNA-loaded microbubbles for lymphatic imaging and lymph node detection after s.c. injection. When taken together with our previous research on transfections with these microbubbles, this paves the way for ultrasound-guided microbubble-assisted drug delivery to intranodal cells. To explore this, we aimed to compare unloaded and mRNA-loaded microbubbles with respects to the lymphatic uptake and contrast enhancement in the lymph nodes upon s.c. injection in dogs.
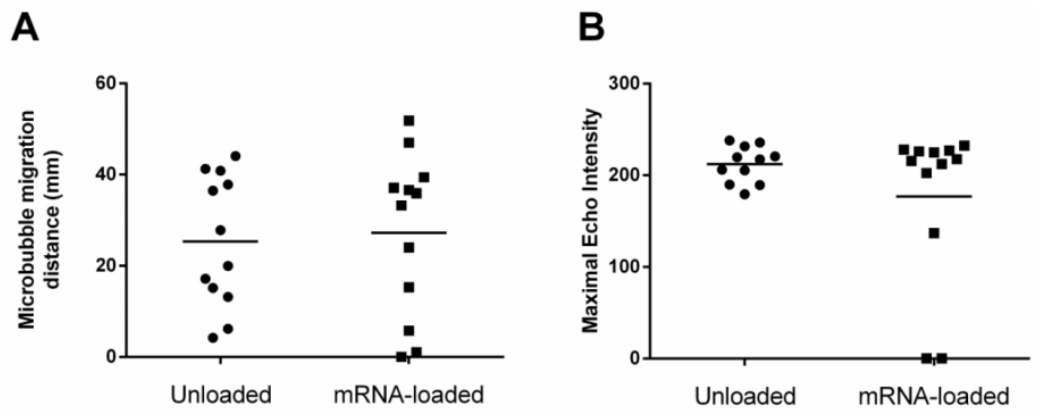

Figure 5: Maximal migration distance and maximal echo intensity of mRNA-loaded and unloaded microbubbles. Scatterplots show (A) the linear migration distance of unloaded and mRNA-loaded microbubbles from the injection site and (B) the maximal echo intensities within the lymphatics for both microbubble types. For both parameters, no statistically significant differences between unloaded and mRNA-loaded microbubbles could be detected.

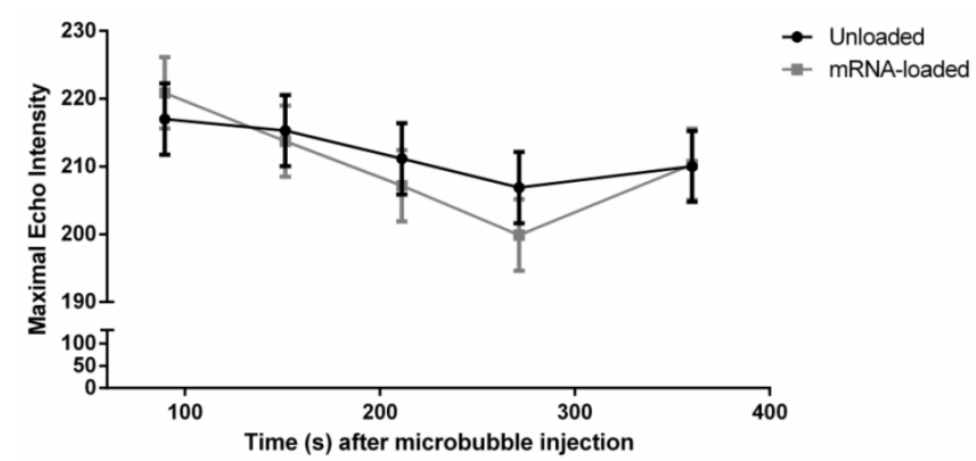

Figure 6: Intralymphatic stability of mRNA-loaded and unloaded microbubbles. The maximal echo intensity was followed as a function of time for both unloaded and mRNA loaded microbubbles. A slow reduction in maximal echo intensity was observed over the 6 min imaging period. No significant differences could be detected between unloaded and mRNA-loaded microbubbles. 

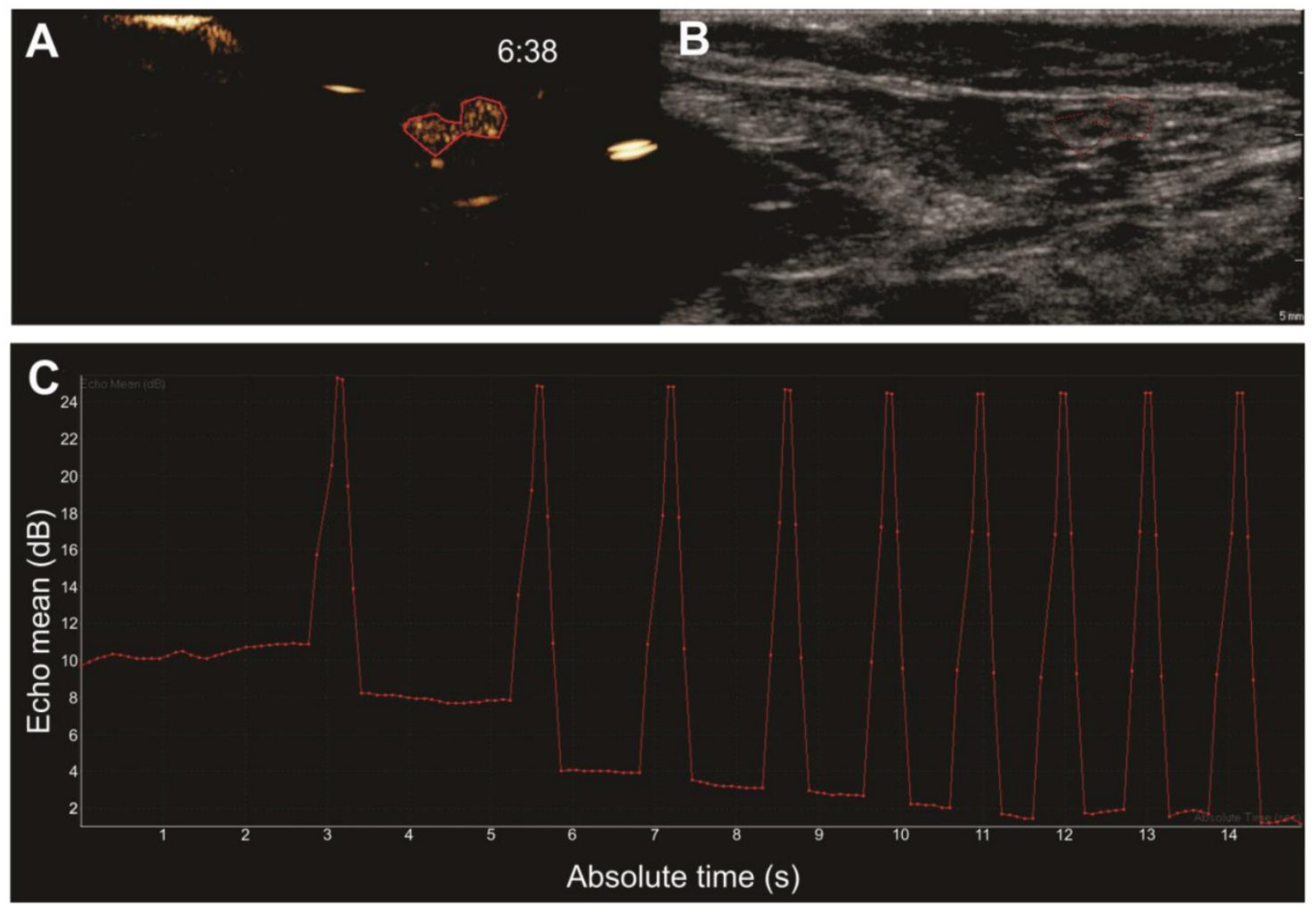

Figure 7: Burst destruction of intranodal microbubbles. After visualization of unloaded microbubble drainage, the scanner's burst function was used to destroy the microbubbles within the lymphatics. Images show (A) CEUS image with a ROI drawn around a lymph node filled with mRNA-loaded microbubbles and (B) the corresponding $\mathrm{B}$-mode image. Burst analysis is represented in $(\mathrm{C})$ as the echo mean $(\mathrm{dB})$, where each burst is visible as an echo mean peak, followed by a reduction of the contrast echo mean. Time after mRNA-loaded microbubble injection is noted on the CEUS images (in min:s).

The lipid microbubbles used in this study could be efficiently loaded with mRNA-lipoplexes, and had mean diameters around $2.5 \mu \mathrm{m}$, which is comparable to the size of commercially available microbubbles. Lipoplex-loading did not significantly alter the mean microbubble diameter. Our imaging results indicated that despite animal-related variation, both types of microbubbles could be detected within the lymph nodes within $1 \mathrm{~min}$ after s.c. injection around the mammary glands. How exactly the microbubbles are transported from the interstitium to the lymph vessels remains controversial, as it goes against the current view of ideal particle properties for lymphatic uptake. It is generally considered that particles between 10 and $100 \mathrm{~nm}$ in size result in the best lymphatic uptake [27]. Although the appearance of $1 \mu \mathrm{m}$ particles in the draining lymph nodes was also observed, their uptake efficiency and speed was significantly reduced and a large fraction of the injected particles were retained at the injection site [30]. In the case of our microbubbles we could observe fast and extensive microbubble uptake into the lymph vessels and draining nodes, despite their larger size. The rapid uptake of microbubbles into the lymphatic system is not well understood. A first explanation could be found within the other physicochemical properties of the microbubble contrast agents we injected. Besides particle size, lipo- philicity and the presence of surface modifications that give stealth properties to injected particles, were also reported to positively impact their lymphatic drainage [30]. Therefore, the presence of the lipid coat in both the microbubbles and the mRNA-lipoplexes could aid to promote microbubble uptake into the lymph vessels. In addition, the microbubbles as well as the mRNA lipoplexes contain lipids that are conjugated to polyethylene glycol (PEG, $15 \mathrm{~mol} \%$ and $2.5 \mathrm{~mol}$ \% for bubbles and lipoplexes respectively), which is known to protect the particles against uptake by phagocytes, thus reducing their premature clearance, resulting in enhanced drainage to the lymphatics. On the other hand, Goldberg et al. proposed a cell-mediated uptake mechanism after s.c. injection of Sonazoid ${ }^{\mathrm{TM}}$ in pigs [6]. They performed electron microscopy on isolated lymph nodes after contrast agent injection, and observed the presence of vacuoles in the intranodal phagocytic cells, which could imply that the microbubbles are first ingested by cells, which then transport them from the interstitium to the lymph vessels and draining lymph nodes. However, this cellular transport through the lymphatics is only likely for microbubbles that are easily phagocytosed. A study by Yanagisawa pointed out that microbubble phagocytosis by primary liver cells was highly dependent on the microbubble composition. Indeed, the 
authors demonstrated that over $99 \%$ of the Sonazoid microbubbles were rapidly phagocytosed by primary liver cells, which can likely be attributed to their shell composition [31]. Sonazoid ${ }^{\mathrm{TM}}$ consists of egg phosphatidyl serine, which is recognized by macrophages and thus enhances phagocytic uptake [32, 33]. In contrasts, anionic Imavist ${ }^{\mathrm{TM}}$ microbubbles (where the anionic charges reduce microbubble contact with cell membranes) and PEGylated SonoVue ${ }^{\mathrm{TM}}$ microbubbles were merely for $0 \%$ or $7.3 \%$ phagocytosed, respectively. As our microbubbles are also PEGylated (by inclusion of 15\% DSPE-PEG (3400)-biotin), and no specific targets for macrophage recognition are present, it is unlikely that our microbubbles were carried into the lymphatics inside of cells.

Upon s.c. injection, we could observe clear migration of both unloaded and mRNA-loaded microbubbles from the injection site into the lymph vessels and the draining lymph nodes. Moreover, our CEUS images nicely correspond to previous reports on the lymphatic drainage of microbubbles, which indicates that the observed structures were indeed lymph nodes [5-7, 34, 35]. The imaging options that are associated with these mRNA-loaded microbubbles could have implications for mRNA delivery as well as for diagnostic purposes. Firstly, the mRNA-loaded microbubbles could provide information on the anatomical features of draining lymph nodes. To our knowledge, this is the first report where CEUS was shown to discriminate between the two different types of connection of lymph vessels to lymph nodes (i.e. the "hollow" nodes and the "filled" lymph nodes) [29]. The impact of this might be dual. First of all, from our perspective of future mRNA delivery to intranodal DCs, it would be a major advantage to be able to discriminate between situations where the afferent lymph vessels discharge the mRNA-loaded bubbles within the core of the lymph node ("filled" nodes) versus lymph vessels that merely run over the surface of the lymph nodes, thus bypassing the actual node, resulting in the absence of a microbubble signal in the center of the node ("hollow" nodes). Only in the first scenario will the mRNA-loaded bubbles be able to reach the intranodal DCs, and is DC transfection with mRNA via sonoporation possible. Therefore, delivery of high-intensity ultrasound pulses to induce microbubble implosion and mRNA delivery should only be performed in the lymph nodes that exhibit the "filled" appearance. Other than for ultrasound-guided drug delivery, this anatomical information could also have benefits with regards to SLN detection. In a tumor setting, the SLN will be the first lymph node that encounters material that directly originates from the tumor, such as disseminating tumor cells. However, if the first lymph node after the tumor has the "hollow" characteristics, this means that the tumor cells do not enter within the node, making it less likely that this node would be populated with tumor cells [29]. Therefore, lymph node biopsies to detect lymph node metastasis are best performed in the first node in which the content of the tumor-draining lymph vessels are actually discharged. CEUS-guided identification of the first "filled" SLN could therefore have an added value to reduce chance of false negatives in SLN biopsies. Of note, other imaging possibilities such as 3D CEUS imaging, could enable improved visualization of the lymphatic drainage and improve variability in measurements.

When comparing mRNA-loaded microbubbles with unloaded microbubbles, we could not observe statistically significant differences with regards to migration distance, mean contrast intensity and the stability of both contrast agents in the lymphatics. As the size distribution of both types of bubbles did not significantly differ, this was not entirely unexpected. In addition, experiments on similar nanoparticle-loaded lipid microbubbles by Luan et al. demonstrated that the shell elasticity of individual unloaded and liposome-loaded microbubbles was nearly the same [36]. The effects of liposome-loading that was observed by the authors were mainly on the microbubble shell viscosity (which was higher for liposome-loaded microbubbles) and thus on the ultrasound-induced microbubble vibrations. Taken together, the impact on these acoustical differences between two types of bubble populations is expected to be limited with respect to contrast-enhancement. However, it is likely that for molecular imaging and therapeutic purposes, which often involve only a single microbubble or a few microbubbles in a given volume, these differences will need to be addressed: liposome-loaded microbubbles exhibited a higher pressure threshold for microbubble vibration, which might indicate that the pressures that will be needed to implode these bubbles in order to locally deposit the mRNA, also need to be higher. This could already be expected based on the burst-destruction of the intranodal mRNA-loaded microbubbles. Using the preset "burst" function on the clinical scanner, the microbubble contrast signal is maximally reduced by $50 \%$, indicating an incomplete intranodal microbubble destruction. Only after 6 bursts the mean echo reaches a minimum. On this basis, the transducer that was used in this study (12-5 MHz linear) is likely not ideal for microbubble destruction that could allow effective sonoporation and mRNA transfection. Other clinical transducers that can emit lower frequencies, which are closer to the resonance frequency of the microbubbles and can emit higher powers, could be 
better suited. In any case, optimization of the various ultrasound parameters such as acoustic pressure, pulse duration and number of pulses, will be required for this purpose [13].

\section{Conclusion}

In conclusion, we were able to show that homemade unloaded as well as mRNA-loaded microbubbles efficiently reach the lymph vessels and nodes upon subcutaneous injection in dogs. Loading of the microbubbles with mRNA-lipoplexes had no significant effect on the distance of microbubble migration from the injection site, nor on the intensity of the observed contrast signals. This shows that theranostic mRNA-loaded microbubbles could have potential for the ultrasound-guided, ultrasound-triggered intranodal delivery of mRNA. However, further research is needed for the optimization of acoustic parameters to most effectively sonoporate target cells of interest.

\section{Abbreviations}

CEUS: contrast-enhanced ultrasound imaging; DC: dendritic cell; SLN: sentinel lymph node; DOTAP: 1,2-dioleoyl-3-trimethylammonium-propane; DOPE: 1,2-dioleoyl-sn-glycero-3-phosphoethanolamine: DSPE: 1,2-distearoyl-sn-glycero-3-phosphoethanolamine; PEG: polyethylene glycol; DPPC: 1,2-dipalmitoyl-sn-glycero-3-phosphocholine; ROI: region of interest; REML: restricted maximum likelihood procedure; $\mathrm{CI}$ : confidence interval; $\mathrm{MI}$ : mechanical index.

\section{Supplementary Material}

Additional File 1:

Supplementary Video 1: CEUS imaging of a vast lymphatic network in an intact female dog. After s.c. injection of unloaded microbubbles around the mammary glands of an intact female dog, a vast lymphatic network could be observed with CEUS imaging. http://www.thno.org/v05p0097s1.mp4

Additional File 2:

Supplementary Video 2: CEUS imaging of 2 lymph vessels connected to one lymph node. Images were recorded after s.c. injection of mRNA-loaded microbubbles in an intact female dog.

http://www.thno.org/v05p0097s2.mp4

Additional File 3:

Supplementary Video 3: CEUS of mRNA-loaded microbubbles 6 min after mRNA-loaded microbubble injection. Images were recorded $6 \mathrm{~min}$ after injection of mRNA-loaded microbubbles in a spayed female dog.

http://www.thno.org/v05p0097s3.mp4
Additional File 4:

Supplementary Video 4: Burst destruction of intranodal microbubbles. After injection of unloaded microbubbles in an intact female dog, multiple bursts were delivered to destroy the intranodal microbubbles and reduce the mean echo intensity. http://www.thno.org/v05p0097s4.mp4 Additional File 5:

Figures S1-S2.

http://www.thno.org/v05p0097s5.pdf

\section{Acknowledgements}

Heleen Dewitte is a doctoral fellow of the Institute for the Promotion of Innovation through Science and Technology in Flanders, Belgium (IWT-Vlaanderen). Ine Lentacker and Katrien Vanderperren are postdoctoral fellows of the Research Foundation-Flanders, Belgium (FWO-Vlaanderen). This project was funded through the FWO grant G016513N. The authors would also like to thank Prof.dr. Jean-Paul Remon for providing the animals, and Prof.dr. Mike Averkiou and Ying Luan for the helpful discussions.

\section{Competing Interests}

The authors have declared that no competing interest exists.

\section{References}

1. Lindner JR. Microbubbles in medical imaging: current applications and future directions. Nat Rev Drug Discov. 2004; 3: 527-532.

2. Sever A, Jones S, Weeks J, et al. Successful outpatient identification of the sentinel lymph node with ultrasound using microbubbles in breast cancer patients: final results. Brit J Surg. 2010; 97: 28-28.

3. Sever AR, Mills P, Jones SE, et al. Preoperative Sentinel Node Identification With Ultrasound Using Microbubbles in Patients With Breast Cancer. Am J Roentgenol. 2011; 196: 251-256.

4. Sever AR, Mills $\mathrm{P}$, Jones $\mathrm{SE}$, et al. Sentinel node identification using microbubbles and contrast-enhanced ultrasonography. Clin Radiol. 2012; 67: 687-694.

5. Goldberg BB, Merton DA, Liu JB, et al. Contrast-enhanced ultrasound imaging of sentinel lymph nodes after peritumoral administration of Sonazoid in a melanoma tumor animal model. J Ultrasound Med. 2011; 30: 441-53.

6. Goldberg BB, Merton DA, Liu JB, et al. Contrast-enhanced sonographic imaging of lymphatic channels and sentinel lymph nodes. J Ultrasound Med. 2005; 24: 953-65.

7. Goldberg BB, Merton DA, Liu JB, et al. Sentinel lymph nodes in a swine model with melanoma: contrast-enhanced lymphatic US. Radiology. 2004; 230: 727-34.

8. Chakera AH, Drzewiecki KT, Ingvar C, et al. Sentinel node imaging. Curr Med Imaging Rev. 2006; 2: 341-346.

9. Swartz MA, Skobe M. Lymphatic function, lymphangiogenesis, and cancer metastasis. Microsc Res Techniq. 2001; 55: 92-99.

10. Keshtgar MR, Ell PJ. Sentinel lymph node detection and imaging. Eur J Nucl Med. 1999; 26: 57-67.

11. Yang WT, Goldberg BB. Microbubble Contrast-Enhanced Ultrasound for Sentinel Lymph Node Detection: Ready for Prime Time? Am J Roentgenol. 2011; 196: 249-250.

12. Zhang F, Niu G, Lu GM, et al. Preclinical Lymphatic Imaging. Mol Imaging Biol. 2011; 13: 599-612

13. Lentacker I, De Cock I, Deckers R, et al. Understanding ultrasound induced sonoporation: Definitions and underlying mechanisms. Adv Drug Deliver Rev. 2014; 72C: 49-64.

14. Xie A, Belcik T, Qi Y, et al. Ultrasound-mediated vascular gene transfection by cavitation of endothelial-targeted cationic microbubbles. JACC Cardiovasc Imaging. 2012; 5: 1253-62.

15. Nomikou $\mathrm{N}$, Tiwari $\mathrm{P}$, Trehan $\mathrm{T}$, et al. Studies on neutral, cationic and biotinylated cationic microbubbles in enhancing ultrasound-mediated gene delivery in vitro and in vivo. Acta Biomater. 2012; 8: 1273-80. 
16. Geers B, Dewitte H, De Smedt SC, et al. Crucial factors and emerging concepts in ultrasound-triggered drug delivery. Journal of controlled release : official journal of the Controlled Release Society. 2012; 164: 248-55.

17. De Temmerman ML, Dewitte H, Vandenbroucke RE, et al. mRNA-Lipoplex loaded microbubble contrast agents for ultrasound-assisted transfection of dendritic cells. Biomaterials. 2011; 32: 9128-9135.

18. Dewitte H, Van Lint $S$, Heirman $C$, et al. The potential of antigen and TriMix sonoporation using mRNA-loaded microbubbles for ultrasound-triggered cancer immunotherapy. J Control Release. 2014; 194: 28-36.

19. Palucka K, Banchereau J. Dendritic-Cell-Based Therapeutic Cancer Vaccines. Immunity. 2013; 39: 38-48.

20. Boczkowski D, Nair SK, Snyder D, et al. Dendritic cells pulsed with RNA are potent antigen-presenting cells in vitro and in vivo. J Exp Med. 1996; 184: $465-472$.

21. Gilboa E, Vieweg J. Cancer immunotherapy with mRNA-transfected dendritic cells. Immunol Rev. 2004; 199: 251-263.

22. Small EJ, Schellhammer PF, Higano CS, et al. Placebo-controlled phase III trial of immunologic therapy with sipuleucel-T (APC8015) in patients with metastatic, asymptomatic hormone refractory prostate cancer. J Clin Oncol. 2006; 24: 3089-3094.

23. Tacken PJ, de Vries IJM, Torensma R, et al. Dendritic-cell immunotherapy: from ex vivo loading to in vivo targeting. Nat Rev Immunol. 2007; 7: 790-802.

24. Caminschi I, Maraskovsky E, Heath WR. Targeting Dendritic Cells in vivo for Cancer Therapy. Front Immunol. 2012; 3: 13.

25. Henri S, Vremec D, Kamath A, et al. The dendritic cell populations of mouse lymph nodes. J Immunol. 2001; 167: 741-748.

26. Randolph GJ, Angeli V, Swartz MA. Dendritic-cell trafficking to lymph nodes through lymphatic vessels. Nat Rev Immunol. 2005; 5: 617-628.

27. Oussoren C, Storm G. Liposomes to target the lymphatics by subcutaneous administration. Adv Drug Deliver Rev. 2001; 50: 143-156.

28. Patsikas MN, Karayannopoulou M, Kaldrymidoy E, et al. The lymph drainage of the neoplastic mammary glands in the bitch: A lymphographic study. Anat Histol Embryol. 2006; 35: 228-234.

29. Tanis PJ, Nieweg OE, Olmos RAV, et al. Anatomy and physiology of lymphatic drainage of the breast from the perspective of sentinel node biopsy. J Am Coll Surg. 2001; 192: 399-409.

30. Swartz MA. The physiology of the lymphatic system. Adv Drug Deliver Rev. 2001; 50: 3-20.

31. Yanagisawa K, Moriyasu F, Miyahara T, et al. Phagocytosis of ultrasound contrast agent microbubbles by Kupffer cells. Ultrasound Med Biol. 2007; 33: 318-325.

32. Callahan MK, Williamson P, Schlegel RA. Surface expression of phosphatidylserine on macrophages is required for phagocytosis of apoptotic thymocytes. Cell Death Differ. 2000; 7: 645-653.

33. Sontum PC. Physicochemical characteristics of Sonazoid (TM), a new contrast agent for ultrasound imaging. Ultrasound Med Biol. 2008; 34: 824-833.

34. Gelb HR, Freeman LJ, Rohleder JJ, et al. Feasibility of Contrast-Enhanced Ultrasound-Guided Biopsy of Sentinel Lymph Nodes in Dogs. Vet Radiol Ultrasoun. 2010; 51: 628-633.

35. Lurie DM, Seguin BD, Schneider PD, et al. Contrast-assisted ultrasound for sentinel lymph node detection in spontaneously arising canine head and neck tumors. Invest Radiol. 2006; 41: 415-421.

36. Luan Y, Faez T, Gelderblom E, et al. Acoustical Properties of Individual Liposome-Loaded Microbubbles. Ultrasound Med Biol. 2012; 38: 2174-2185. 